\section{En los servicios de medicina intensiva se debe solicitar consentimiento informado para la transfusión no urgente}

\section{Sr. Director:}

La Ley General de Sanidad ${ }^{1}$ reguló que el consentimiento se hará «por escrito» en procedimientos terapéuticos invasores. Posteriormente, la ley $41 / 2002^{2}$ estableció que el consentimiento informado se otorgará por escrito en los casos siguientes: intervención quirúrgica, procedimientos diagnósticos y terapéuticos invasores y, en general, cuando se vaya a realizar procedimientos que supongan riesgos o inconvenientes de notoria y previsible repercusión negativa en la salud del paciente. Se puede considerar la transfusión no urgente como un procedimiento terapéutico invasor del que debe informarse al paciente o en su caso a la familia y pedirle su autorización.

El grupo de bioética de la SEMICYUC, elaboró unas recomendaciones sobre el consentimiento informado en los servicios de medicina intensiva (SMI) que fueron publicadas en su órgano de expresión, la revista Medicina InTEnsiva ${ }^{3}$. En síntesis, las conclusiones del grupo eran rechazar el consentimiento genérico por la dificultad de documentar toda la complejidad del procedimiento en los SMI y apoyar el consentimiento por procedimientos como traqueostomía, transfusión sanguínea no urgente, fibroscopia, intervención quirúrgica urgente, hemodiálisis, marcapasos que no sea de urgencia vital, plasmaféresis, angioplastia, nuevas técnicas o aquellas de las que aún no se ha demostrado eficacia. Se enfatizaba también la necesidad de anotar en la historia clínica todos los aspectos más relevantes de la información que cada día se aporta al paciente o a sus familiares.

Se aconseja solicitar el consentimiento antes de la primera transfusión no urgente; en el caso de tener que realizar más al mismo paciente, no es necesario un consentimiento para cada transfusión, aunque es recomendable anotar en la historia clínica que se informó previamente.

En junio del presente año, se realizó una encuesta telefónica en 15 SMI para evaluar el seguimiento de estas recomendaciones. Todas ellas seguían en líneas generales las recomendaciones de la SEMICYUC, salvo en el caso de la transfusión sanguínea, que siempre fue considerada como urgente y por lo tanto exenta de la obligación de realizarla tras el proceso de obtención del consentimiento informado.

El Grupo de Bioética de la SEMICYUC considera que existe un efecto de «arrastre» debido a que a menudo las indicaciones de transfusión en los SMI son urgentes, por lo que en algunas situaciones se obvia la solicitud del consentimiento informado para la transfusión no urgente, a pesar de ser un imperativo legal, cuyo cumplimiento recordamos.

Es necesario conocer las obligaciones reguladas por la legislación vigente, como un primer paso para que todos los procedimientos realizados en los SMI se efectúen en el contexto de una práctica clínica que aspira a la excelencia.

J.F. SOLSONA DURÁN ${ }^{\mathrm{a}}$, I. SARALEGUI RETA ${ }^{\mathrm{b}}$ Y GRUPO DE BIOÉTICA DE LA SEMICYUC

${ }^{a}$ Servicio de Medicina Intensiva. Hospital del Mar. Barcelona. España.

${ }^{b}$ Servicio de Medicina Intensiva. Hospital San Millán. Logroño. La Rioja. España.

\section{BIBLIOGRAFÍA}

1. Ley General de Sanidad, del 25 de abril de 1986. Artículo 8.2. BOE, 29-4-1986.

2. Ley $41 / 2002$, de 14 de noviembre, básica reguladora de la autonomía del paciente y de derechos y obligaciones en materia de información y documentación clínica. Capítulo IV, Artículo 8.

3. Solsona JF, Cabré L, Abizanda R, Campos JM, Sainz A, Martín MC, et al; grupo de Bioética de la SEMICYUC. Recomendaciones del grupo de Bioética de la SEMICYUC sobre el Consentimiento Informado en UCI. Med Intensiva. 2002;26:254-5. 